\title{
DEVELOPMENT OF SIMPLE AND SENSITIVE SCORE TO ASSESS THE RISK OF PNEUMONIA IN COVID-19 PATIENTS
}

\author{
Macarena Hormanstorfer ${ }^{1 *}$, Martin A. Ragusa ${ }^{1}$, Lucia Poggio ${ }^{1}$, Jorge Moreira-Facundo ${ }^{1}$, \\ Zulma Orellana-Villa ${ }^{1}$, Florencia A. Bobrowski ${ }^{1}$, Joaquin Martinez-Serventi ${ }^{1}$, Santiago N. \\ Piombi Adanza ${ }^{1}$, Jose A. Enrique Barletta ${ }^{2}$, Alicia Sisto ${ }^{2}$, Hugo Delle-Piane ${ }^{1}$, Juan M. Carrillo ${ }^{1}$, \\ Jose L. Presas ${ }^{1}$, and Francisco Paulin ${ }^{1}$ \\ Divisions of ${ }^{1}$ Internal Medicine and ${ }^{2}$ Infectious Diseases, Hospital Juan A. Fernández, Buenos Aires, Argentina
}

\begin{abstract}
Background: Severe pneumonia is the most common cause of intensive care unit (ICU) admission and death due to novel coronavirus (SARS-CoV-2) respiratory disease (COVID-19). Due to its rapid outbreak, units for the evaluation of febrile patients in the pre-hospital setting were created. Objective: The objective of the study was to develop a sensitive and simple tool to assess the risk of pneumonia in COVID-19 patients and thus select which patients would require a chest imaging study. Materials and Methods: We conducted a cross-sectional study in a cohort of individuals with suspected COVID-19 evaluated in a public academic healthcare center in Buenos Aires city. All adult patients with positive RT-PCR assay for SARS-COV2 between April 24 and May 19 of 2020 were included in the study. Pneumonia was defined as the presence of compatible signs and symptoms with imaging confirmation. Univariate and multivariate logistic regression was performed. A risk indicator score was developed. Results: One hundred and forty-eight patients were included, 71 (48\%) received the diagnosis of pneumonia. The final clinical model included four variables: age $\geq 40$ years, cough, absence of sore throat, and respiratory rate $\geq 22$. To create the score, we assigned values to the variables according to their ORs: 2 points for respiratory rate $\geq 22$ and 1 point to the other variables. The AUC of the ROC curve was 0.80 ( $\mathrm{Cl} 95 \% 0.73-0.86$ ). A cutoff value of 2 showed a sensitivity of $95.7 \%$ and a specificity of $43.24 \%$. Conclusion: This sensible score may improve the risk stratification of COVID-19 patients in the pre-hospital setting. (REV INVEST CLIN. 2021;73(1):52-8)
\end{abstract}

Key words: COVID-19. SARS-CoV-2. Pneumonia. Risk indicator score.

\section{INTRODUCTION}

In December 2019, a novel coronavirus was identified as the cause of a cluster of pneumonia cases in $\mathrm{Wu}$ han, a city in the Hubei province of China. Rapidly, in
January 2020, Chinese scientists sequenced the genome of the novel coronavirus designated SARS$\mathrm{CoV} 2^{1}$. This agent has been found to be responsible for several cases of severe acute respiratory syndrome around the world. The coronavirus disease (COVID-19)
*Corresponding author:

Macarena Hormanstorfer

E-mail: hormanstorferm@gmail.com
Received for publication: 23-06-2020

Approved for publication: 18-08-2020

DOI: $10.24875 / R I C .20000295$

0034-8376 / (c) 2020 Revista de Investigación Clínica. Published by Permanyer. This is an open access article under the CC BY-NC-ND license (http://creativecommons.org/licenses/by-nc-nd/4.0/). 
was declared a Public Health Emergency of International Concern (PHEI) on January 30, 2020, and was defined as pandemic on March 11, 2020. At the end of July, the WHO reported around 17.106.007cases of COVID-19 and 668.910 deaths $^{2}$. Despite public health efforts aimed to control the disease, numerous health-care systems have collapsed because of COVID-193,4. On March 3, 2020, the first case was confirmed in Argentina. Almost 3 months later, the total number of COVID-19 cases is 191.302. Most of them reside in Buenos Aires ${ }^{5}$.

Argentina is a developing country where a significant proportion of the population receives medical attention in the public health-care system. Due to the rapid spread of this infectious disease, and the imminent collapse of public health-care system, health and government authorities implemented measures to slow the spread of the disease and guarantee medical assistance to suspected cases. Twenty Febrile patients' units (FPU) were created to expand patient care capacity. There, suspected cases are identified, and the tests are performed. As these units are close to hospitals, they also make triage admission and define which patients require emergency department evaluation, according to their severity. The Fernandez Hospital is an academic public healthcare center located in Buenos Aires city, designated to give attention to suspected and confirmed cases of COVID-19.

Epidemiological and clinical characteristics of patients with COVID-19 have been reported in several international studies, most of them from China. Those studies described the spectrum of disease severity, which includes an asymptomatic course, or mildly symptomatic upper respiratory tract illness, to severe viral pneumonia with radiological opacities, respiratory failure, and death 6 . Approximately $15 \%$ of the infected patients will develop severe disease and require hospitalization, and $5 \%$ will require admission to an intensive care unit ${ }^{7}$. The most common reported reason for requiring intensive care admission has been the need of respiratory support ${ }^{6}$. Patients who require ICU admission tend to be older and have a higher prevalence of comorbid conditions such as hypertension and diabetes, among others ${ }^{6,8,9}$. Regarding radiological findings, patients with COVID-19 showing lung opacities compatible with pneumonia may have a worse prognosis than those without them ${ }^{10}$. That group of patients may double the odds of progression to severe disease, compared with patients with normal chest image ${ }^{11-14}$.

Clinical prediction rules are a useful resource to know which patients are at risk of having a specific outcome. The knowledge of isolated risk factors is often not sufficient to determine the probability of having a specific event. To the best of our knowledge, rules to assess the risk of pneumonia in COVID-19 patients are lacking. A simple and sensible clinical rule to assess the risk of pneumonia composed by clinical and demographic features collected at the patient first assessment could be a useful tool in the pre-hospital setting to rule out the diagnosis of pneumonia without the realization of a chest image. The aim of this observational cross-sectional study was to develop that rule.

\section{MATERIALS AND METHODS}

We conducted a cross-sectional study based on the analysis of a prospective cohort of individuals with suspected COVID-19 admitted to a general ward after being initially evaluated at febrile patients' units (FPU) and emergency department (ED) of Fernandez Hospital, in Buenos Aires city. All patients who met the definition of suspected case were tested. If the first test was negative and the patient had compatible signs, symptoms, or chest images findings, a second test was performed. Patients with the diagnosis of other acute respiratory disease were excluded from the study. All adult patients with positive RT-PCR assay for SARS-CoV-2 in nasal or pharyngeal swab evaluated between April 24 and May 19 of 2020 were included in the study. The following data were consigned in a structured form during the patient's first evaluation: epidemiological and demographic data, comorbidities (diabetes, obesity, HIV infection, chronic kidney disease, alcohol abuse, COPD, asthma, smoking history, tuberculosis infection, hypertension, congestive heart failure, and coronary disease), use of angiotensin-converting enzyme inhibitors (ACEls) and angiotensin receptor blockers (ARBs), new signs and symptoms at disease onset, vital sings at the ED or the FPU (blood pressure, temperature, heart rate, respiratory rate, and oxygen saturation), modified NEWS score, qSOFA and CURB 65 scores, blood tests, and chest images. A member of the data management team and review by a second member of that 
team charged data submitted by physicians on paper forms in a digital database. Clinical manifestations related to chronic conditions (i.e., COPD) were not considered. The diagnosis of pneumonia was defined as the presence of compatible signs and symptoms (fever with cough or difficult breathing) with imaging confirmation. All patient included has at least one of the following chest images to make a pneumonia diagnosis: chest $\mathrm{X}$-ray (usually made at ED), lung ultrasound (usually made at FPU), or chest CT (performed in patients in whom the diagnosis of pneumonia was still not clear after the realization of X-ray or lung ultrasound).

\section{Statistical analysis}

Comparisons between groups were made using a t-test, Mann-Whitney test, and Chi-square. Univariate logistic regression was performed, with the presence of pneumonia as a dependent variable. Continuous variables were categorized according to cutoff points defined by receiver operating curve analysis. Statistical significance was analyzed with Wald test. Variables with a $p<0.1$ were considered candidates for the multivariate analysis. The decision to include a variable in the definitive model was guided with the likelihood ratio test. We prioritized those variables which can be easily measured without medical equipment. Clinical significance was defined with a $p<0.05$. The HosmerLemeshow goodness of fit test was used to evaluate the model calibration. A risk indicator score was developed and values were assigned to the included variables according to regression coefficients and their corresponding odd ratios. The discrimination accuracy of the score was expressed as the area under the receiver operating curve. Regarding the sample size, minimum of 50 cases had to be included to have enough power to build a score with five risk factors, according to the "one variable per ten events" rule ${ }^{15}$. We performed an internal validation of the model with the Bootstrap method. The manuscript was written according to the STROBE initiative for the communication of observational studies ${ }^{16}$. The Institutional Ethics Committee of our hospital reviewed and approved the study.

\section{RESULTS}

Between April 24 and May 19 of 2020, 348 patients with suspected COVID-19 were admitted in the
Fernandez Hospital, derived from the ED or the FPU. Of them, 200 had a negative test for SARS-CoV-2 and were excluded from the study. Baseline characteristics of patients with a positive test for SARS-CoV-2 $(n=148)$ are presented in Table 1 . The mean (SD) age was 42.7 years (16.5), with a predominance of male gender $(56.7 \%)$. The most common symptoms at baseline were fever (66.9\%), headache ( $56 \%)$, and cough ( $62 \%)$. A total of 71 patients ( $48 \%$ ) received the diagnosis of pneumonia.

The comparison between groups according to the presence or absence of pneumonia is shown in Table 1 . Patients with pneumonia were older and more likely to present fever, cough, dyspnea, and thoracic pain. Those patients without pneumonia were more likely to have a sore throat. Patients with pneumonia had a higher temperature, respiratory rate and NEWS score, and lower oxygen saturation. Regarding laboratory findings, lymphopenia, elevated $\mathrm{C}$ reactive protein, and lactate dehydrogenase were more frequent in pneumonia patients. We found no difference between groups in gender, comorbidities, qSOFA, and CURB-65 scores.

Regarding the lung images of patients with pneumonia, 34 patients ( $47 \%$ ) were diagnosed with $X$-ray and 24 (33\%) with lung ultrasound. In 31 cases (43\%), the diagnosis of pneumonia was achieved or confirmed with a CT scan.

We studied the association between demographic and baseline clinical characteristics and the presence of pneumonia. Eight variables were associated with the diagnosis of pneumonia in univariate analysis (Table 2): age $\geq 40$ years, presence of cough, absence of sore throat, respiratory rate $\geq 22$, temperature $\geq$ $37.5^{\circ} \mathrm{C}$, oxygen saturation $\leq 94 \%$, dyspnea, and male gender. All these variables were included in the initial multivariate analysis. The first model (not shown) was modified according to its statistical performance and clinical applicability.

The final clinical model included four variables: age $\geq 40$ years, cough, absence of sore throat, and respiratory rate $\geq 22$ (Table 3 ). To develop the risk indicator score, we assigned values to the included variables according to their regression coefficients and the corresponding odd ratios. The analysis of the discriminatory capacity of the rule showed an AUC of $0.80(\mathrm{Cl}$ $95 \% 0.73-0.86$ ) (Fig. 1 ). We chose a value of 2 points 
Table 1. General characteristics of patients with confirmed COVID-19 infection admitted in Fernández Hospital between April 24 and May 19 of 2020. Comparison between patients with and without pneumonia

\begin{tabular}{|c|c|c|c|c|}
\hline & $\begin{array}{c}\text { Total } \\
(n=148)\end{array}$ & $\begin{array}{l}\text { Patients with } \\
\text { pneumonia } \\
(n=71)\end{array}$ & $\begin{array}{l}\text { Patients without } \\
\text { pneumonia } \\
(n=77)\end{array}$ & $p$ value \\
\hline \multicolumn{5}{|l|}{ Demographics } \\
\hline Age, mean (SD) & $42.71(16.51)$ & $47.73(14.7)$ & $38.22(16.83)$ & $<0.001$ \\
\hline Male gender, n (\%) & $84(56.7)$ & $46(64.79)$ & $38(49.35)$ & 0.058 \\
\hline \multicolumn{5}{|l|}{ Comorbidities } \\
\hline Obesity, n (\%) & $25(16.89)$ & $15(21.13)$ & $10(12.99)$ & 0.187 \\
\hline Pregnant, n (\%) & $2(1.35)$ & $1(1.3)$ & $1(1.41)$ & 0.954 \\
\hline HIV infection, n (\%) & $4(2.7)$ & $2(2.82)$ & $2(2.6)$ & 0.934 \\
\hline Chronic kidney disease, n (\%) & $1(0.68)$ & $0(0)$ & $1(1.3)$ & 0.335 \\
\hline Current smoker, n (\%) & $13(8.78)$ & $7(9.09)$ & $6(8.45)$ & 0.891 \\
\hline Alcohol abuse, n (\%) & $7(4.73)$ & $1(1.41)$ & $6(7.79)$ & 0.068 \\
\hline COPD, n (\%) & $1(0.68)$ & $1(1.41)$ & $0(0)$ & 0.296 \\
\hline Asthma, n (\%) & $8(5.41)$ & $2(2.82)$ & $6(7.79)$ & 0.181 \\
\hline History of TBC infection, $\mathrm{n}(\%)$ & $6(4.05)$ & $2(2.82)$ & $4(5.19)$ & 0.464 \\
\hline Hypertension, n (\%) & $21(14.19)$ & $13(18.31)$ & $8(10.39)$ & 0.168 \\
\hline Congestive heart failure, n (\%) & $2(1.35)$ & $1(1.41)$ & $1(1.3)$ & 0.954 \\
\hline Coronary disease, n (\%) & $2(1.35)$ & $0(0)$ & $2(2.6)$ & 0.172 \\
\hline ACEIs, n (\%) & $8(5.44)$ & $6(8.57)$ & $2(2.6)$ & 0.111 \\
\hline ARBs, n (\%) & $4(2.72)$ & $3(4.24)$ & $1(1.3)$ & 0.266 \\
\hline \multicolumn{5}{|l|}{ Symptoms } \\
\hline Fever, n (\%) & $97(66.9)$ & $54(76.06)$ & $43(58.11)$ & 0.022 \\
\hline Dyspnea, n (\%) & $43(29.86)$ & $29(41.43)$ & $14(18.92)$ & 0.003 \\
\hline Cough, n (\%) & $90(62.07)$ & $51(71.83)$ & $39(52.7)$ & 0.018 \\
\hline Sore throat, n (\%) & $46(31.72)$ & $13(18.57)$ & $33(44)$ & 0.001 \\
\hline Vomits, n (\%) & $12(8.33)$ & $7(10)$ & $5(6.76)$ & 0.482 \\
\hline Diarrhea, n (\%) & $26(18.06)$ & $15(21.43)$ & $11(14.86)$ & 0.306 \\
\hline Myalgias, n (\%) & $49(33.79)$ & $25(35.21)$ & $24(32.43)$ & 0.724 \\
\hline Arthralgias, n (\%) & $20(13.89)$ & $13(18.57)$ & $7(9.56)$ & 0.114 \\
\hline Malaise, n (\%) & $66(45.21)$ & $36(50.7)$ & $30(40)$ & 0.194 \\
\hline Headache, n (\%) & $82(56.16)$ & $38(54.29)$ & $44(57.89)$ & 0.661 \\
\hline Conjunctival injection, n (\%) & $9(6.29)$ & $2(2.9)$ & $7(9.46)$ & 0.106 \\
\hline Chest pain, n (\%) & $16(11.11)$ & $12(17.14)$ & $4(5.41)$ & 0.025 \\
\hline Confusion, n (\%) & $1(0.69)$ & $1(1.43)$ & $0(0)$ & 0.302 \\
\hline \multicolumn{5}{|l|}{ Physical examination } \\
\hline $\begin{array}{l}\text { Systolic blood pressure, } \\
\text { mean (SD) }\end{array}$ & $121,63(15.37)$ & $121.88(14.28)$ & $121.4(16.46)$ & 0.854 \\
\hline Temperature, mean (SD) & $36.89(0.97)$ & $37.09(0.97)$ & $36.7(0.94)$ & 0.019 \\
\hline Heart rate, mean (SD) & $92.88(14.98)$ & $94.95(16.02)$ & $90.87(13.71)$ & 0.107 \\
\hline Respiratory rate, mean (SD) & $19.85(3.9)$ & $21.34(4.29)$ & $18.43(3.09)$ & $<0.001$ \\
\hline Oxygen saturation, mean (SD) & $96.15(2.25)$ & $95.18(2.5)$ & $97.11(1.46)$ & $<0.001$ \\
\hline
\end{tabular}


Table 1. General characteristics of patients with confirmed COVID-19 infection admitted in Fernández Hospital between April 24 and May 19 of 2020. Comparison between patients with and without pneumonia (continued)

\begin{tabular}{|c|c|c|c|c|}
\hline & $\begin{array}{c}\text { Total } \\
(n=148)\end{array}$ & $\begin{array}{l}\text { Patients with } \\
\text { pneumonia } \\
\quad(n=71)\end{array}$ & $\begin{array}{l}\text { Patients without } \\
\text { pneumonia } \\
(n=77)\end{array}$ & $\mathrm{p}$ value \\
\hline \multicolumn{5}{|l|}{ Physical examination } \\
\hline $\begin{array}{l}\text { NEWS score at admission, } \\
\text { median (IQR) }\end{array}$ & $2(1-4)$ & $3(2-5)$ & $1(0-3)$ & $<0.001$ \\
\hline $\begin{array}{l}\text { CURB } 65 \text { at admission, } \\
\text { median (IQR) }\end{array}$ & $1(0-1)$ & $1(0-1)$ & $1(0-1)$ & 0.71 \\
\hline $\begin{array}{l}\text { qSOFA at admission, } \\
\text { median (IQR) }\end{array}$ & $0(0-0)$ & $0(0-0)$ & $0(0-1)$ & 0.221 \\
\hline \multicolumn{5}{|l|}{ Blood tests } \\
\hline Platelets, mean (SD) & $213.4(105.4)$ & $215.41(80.95)$ & $211.51(124.78)$ & 0.827 \\
\hline Lymphocytes\%, mean (SD) & $23.91(11.56)$ & $20.66(9.61)$ & $26.97(12.45)$ & 0.001 \\
\hline $\mathrm{RCP}$, median (IQR) & $2.4(0.7-4.84)$ & $4.48(2.93-7.2)$ & $1(0.5-2.2)$ & $<0.001$ \\
\hline LDH, median (IQR) & $239.5(200-304)$ & $272(218-380)$ & $211(198-280)$ & $<0.001$ \\
\hline
\end{tabular}

COPD: chronic obstructive pulmonary disease; TBC: tuberculosis disease; ACEls: angiotensin-converting enzyme inhibitors; ARBs: angiotensin II receptor blockers; $\mathrm{RCP}$ : reactive protein $\mathrm{C}$; $\mathrm{LDH}$ : lactate dehydrogenase.

Table 2. Univariate and multivariate logistic regression analysis with the presence of pneumonia as dependent variable. The adjusted ORs are shown only in variables included in the final model

\begin{tabular}{lcccc}
\hline & Unadjusted OR & Cl 95\% & Adjusted OR & Cl 95\% \\
\hline Age $\geq 40$ years & 4.72 & $2.35-9.48$ & 3.49 & $1.59-7.68$ \\
Cough & 2.28 & $1.14-4.56$ & 3.21 & $1.37-7.5$ \\
Absence of sore throat & 3.44 & $1.61-7.33$ & 3.15 & $1.26-7.86$ \\
Respiratory rate $\geq 22$ & 4.52 & $2.12-9.63$ & - & - \\
Temperature $\geq 37.5$ & 3.01 & $1.49-6.08$ & - & - \\
Dyspnea & 3.03 & $1.42-6.42$ & - & - \\
Male gender & 1.88 & $0.97-3.65$ & - & - \\
Oxygen saturation $\leq 94 \%$ & 19.14 & $4.32-84.78$ & & - \\
\hline
\end{tabular}

Table 3. Variables included in the risk indicator score

\begin{tabular}{lc}
\hline Variable & Points \\
\hline Age $\geq 40$ & 1 \\
Cough & 1 \\
Absence of sore throat & 1 \\
Respiratory rate $\geq 22 \mathrm{pm}$ & 2 \\
\hline
\end{tabular}

as the best cutoff of the rule, with a sensitivity of $95.7 \% \%$ and a specificity of $43.24 \%$. We prioritized sensitivity over specificity because the score was conceived as a potential tool to rule out the diagnosis of pneumonia without the realization of a chest image in a pre-hospital setting.
The calibration accuracy of the final model showed good results (Hosmer-Lemeshow goodness of fit test $\mathrm{p}=0.59$ ). The internal validation with Bootstrap method demonstrated that after 1000 replications, the score showed an AUC of 0.80 ( $\mathrm{Cl} 95 \%$ 0.73-0.86; $\mathrm{p}<0.001$ ).

\section{DISCUSSION}

This study shows that certain clinical and demographic factors (such as age $\geq 40$ years, cough, absence of sore throat, respiratory rate $\geq 22$, temperature $\geq 37.5$, oxygen saturation $\leq 94 \%$, dyspnea, and male gender) are associated with the presence of pneumonia in patients with COVID-19. We created 
Figure 1. Receiver operating characteristic curve of the risk indicator score developed to predict the presence of pneumonia in COVID 19 patients. It shows an AUC of $0.80(\mathrm{Cl} 95 \%$ 0.73-0.86). A cutoff value of 2 points has a sensitivity of $95.7 \% \%$ and a specificity of $43.24 \%$.

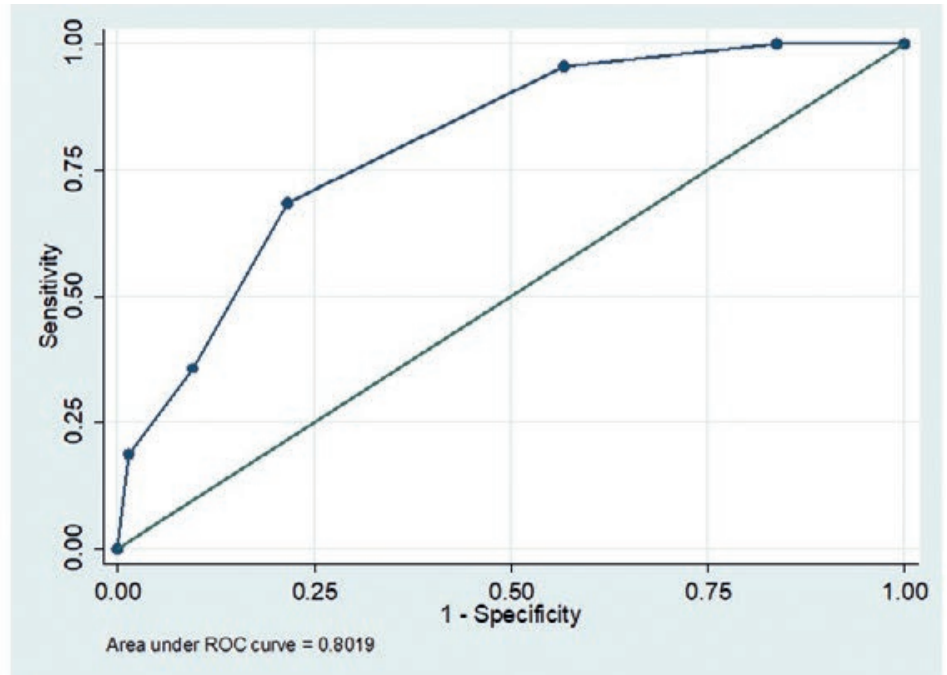

a score to assess the risk of pneumonia in COVID-19 patients based on 4 of these risk factors that showed a good discriminatory capacity. To the best of our knowledge, this is the first risk indicator score developed to identify pneumonia in COVID-19 patients.

Risk factors associated with worse prognostic in patients with COVID-19 pneumonia have been described in several international studies ${ }^{17,18}$. Fei et al. showed in a retrospective multicentric cohort study of 191 patients with COVID-19 pneumonia, that older age (OR $1.1095 \% \mathrm{Cl}$ 1.03-1.17 per year increase; $p=0.0043$ ) higher SOFA score (OR 5.65, $95 \% \mathrm{Cl} 2.61-12.23$; $\mathrm{p}<0.0001$ ), and d-dimer greater than $1 \mathrm{ug} / \mathrm{mL}$ (OR 18.42, 95\% Cl 2.64-128.55; $\mathrm{p}=0.0033$ ) on admission were associated with an increased risk of death during hospitalization ${ }^{8}$. Chaomin et al. reported in a retrospective cohort study of 201 patients with COVID-19 pneumonia that risk factors associated with the development of acute respiratory distress syndrome (ARDS) or death were older age (HR 3.26; 95\% Cl 2.08-5.11; and $\mathrm{HR}, 6.17 ; 95 \% \mathrm{Cl}, 3.26-11.67$, respectively), neutrophilia (HR, 1.14; $95 \% \mathrm{Cl}, 1.09-1.19$; and HR, $1.08 ; 95 \% \mathrm{Cl}, 1.01-1.17$, respectively), and organ dysfunction (higher lactate dehydrogenase [HR, $1.61 ; 95 \% \mathrm{Cl}, 1.44-1.79$ and $\mathrm{HR}, 1.30 ; 95 \% \mathrm{Cl}$,
1.11-1.52, respectively] and D-dimer elevation [HR, 1.03; $95 \% \mathrm{Cl}, 1.01-1.04$ and $\mathrm{HR}, 1.02$; $95 \% \mathrm{Cl}, 1.01$ 1.04, respectively]) $)^{9}$. In addition to these findings, radiological abnormalities in patients with COVID-19 infection have also shown an association with worse prognosis ${ }^{10,19-22}$. A group of authors found that some specific chest CT patterns (such as consolidation or crazy-paving sign) were related to severe disease. Another group of researchers utilized a CT score to calculate the extension of lung opacities and found that patients with higher values of the score had worse prognosis ${ }^{20}$. All this information shows the ability of chest images to detect patients at risk of presenting a severe course of the disease and highlights the relevance of the diagnosis of pneumonia in patients with suspected COVID-19. However, performing a chest image is not easy because the resource is not always available in the pre-hospital setting. A simple score to safely rule out pneumonia in COVID-19 patients could help to reduce the number of suspected cases that need imaging. This may improve the risk stratification in the pre-hospital setting, leaving hospital resources free for patients with a worse prognosis. In summary, we believe that the ability of this score to rule out the presence of lung opacities in the chest images makes it a useful tool in the management of COVID-19 patients. 
Our study has limitations. First, we developed the score with information from admitted patients, but it was conceived to be utilized in a different clinical scenario (the pre-hospital evaluation). This difference may introduce a context bias ${ }^{23}$. Second, the diagnosis of pneumonia was achieved with different methods, depending on the place where the patient was initially evaluated (FPU or ED). In addition, the physicians registering forms with clinical data were not blinded to imaging results. This fact could imply the presence of test review bias. On the other side, the radiologist that interpreted the images may have been aware of clinical data, which could introduce diagnostic review bias ${ }^{23}$. We believe that, although it has limitations, our study has interesting results and may contribute to improve the evaluation of patients with suspected COVID-19 infection, especially in the pre-hospital setting. However, external validation of our score is still needed.

\section{REFERENCES}

1. Phelan AL, Katz R, Gostin LO. The novel coronavirus originating in Wuhan, China: challenges for global health governance. JAMA. 2020;323:709-10

2. World Health Organization. Coronavirus Disease 2019 (COVID-19) Situation Report-193; 2020. Available from: https:// www.who.int/docs/default-source/coronaviruse/situationreports / 20200731 -covid-19-sitrep-193. pdf?sfvrsn=42a0221d 2 .

3. Arabi YM, Murthy S, Webb S. COVID-19: a novel coronavirus and a novel challenge for critical care. Intensive Care Med. 2020;46:833-6.

4. Xie J, Tong Z, Guan X, Du B, Qiu H, Slutsky AS. Critical care crisis and some recommendations during the COVID-19 epidemic in China. Intensive Care Med. 2020;46:837-40.

5. Ministerio de Salud de la Nación. Nuevo Coronavirus COVID-19. Informe Diario Vespertino; 2020. Available from: http://wwww. file:///C:/Users/Nos/Documents/COVID-19\%20HJAF/Predictores\%20neumonia/31-07-20-reporte-vespertino-covid-19. pdf. [Last accessed on 2020 Jul 31].

6. Wang D, Hu B, Hu C, Zhu F, Liu X, Zhang J, et al. Clinical characteristics of 138 hospitalized patients with 2019 novel coronavirus-infected pneumonia in Wuhan, China. JAMA. 2020; 323:1061-9.
7. Epidemiology Working Group for NCIP Epidemic Response, Chinese Center for Disease Control and Prevention. The epidemiological characteristics an outbreak of 2019 novel coronavirus diseases (COVID-19) in China. Zhonghua Liu Xing Bing Xue Za Zhi. 2020;41:145-51.

8. Fei Z, Ting Y, Ronghui D, Fan G, Liu Y, Liu Z, et al. Clinical course and risk factors for mortality of adult inpatients with COVID-19 in Wuhan, China: a retrospective cohort study. Lancet. 2020; 6736:1-9.

9. Wu C, Chen X, Cai Y, Xia J, Zhou X, Xu Z, et al. Risk factors associated with acute respiratory distress syndrome and death in patients with coronavirus disease 2019 pneumonia in Wuhan, China. JAMA Intern Med. 2020;180:1-11.

10. Yuan $M$, Yin $W$, Tao $Z$, Tan $W, H u$ Y. Association of radiologic findings with mortality of patients infected with 2019 novel coronavirus in Wuhan, China. PLoS One. 2020;15:1-10.

11. Chen J, Qi T, Liu L, Ling Y, Qian Z, Li T, et al. Clinical progression of patients with COVID-19 in Shanghai, China. J Infect. 2020; 80:1-6

12. Young BE, Ong SW, Kalimuddin S, Low JG, Tan SY, Loh J, et al. Epidemiologic features and clinical course of patients infected with SARS-CoV-2 in Singapore. JAMA J Am Med Assoc. 2020;323:1488-94

13. Zhang J], Dong $X$, Cao YY, Yuan YD, Yang YB, Yan YQ, et al Clinical characteristics of 140 patients infected with SARSCoV-2 in Wuhan, China. Allergy. 2020;75:1-12.

14. Guan W, Ni Z, Hu Y, Liang W, Ou C, He J, et al. Clinical characteristics of coronavirus disease 2019 in China. N Engl J Med. 2020;382:1708-20.

15. Steyerberg EW, Eijkemans MJ, Harrell FE, Habbema JD. Prognostic modelling with logistic regression analysis: a comparison of selection and estimation methods in small data sets. Stat Med. 2000;19:1059-79.

16. Von Elm E, Altman DG, Egger M, Pocock SJ, Gotzsche PC, Vandenbroucke JP. Directrices para comunicación de estudios observacionales. Gac Sanit. 2008;22:144-50.

17. Wang X, Fang X, Cai Z, Wu X, Gao X, Min J, et al. Comorbid chronic diseases and acute organ injuries are strongly correlated with disease severity and mortality among COVID-19 patients: a systemic review and meta-analysis. Research. 2020; 2020:1-17.

18. Kumar A, Aurora A, Sharma P, Anikhindi S, Bansal N, Singla V, et al. Clinical features of COVID-19 and factors associated with severe clinical course: a systematic review and meta-analysis. Lancet. 2020;607:1-73.

19. Liu KC, Xu P, Lv WF, Qiu XH, Yao JL, Gu JF et al. CT manifestations of coronavirus disease-2019: a retrospective analysis of 73 cases by disease severity. Eur J Radiol. 2020;126;108941.

20. Zhao W, Zhong Z, Xie X, Yu Q, Liu J. Relation between chest CT findings and clinical conditions of coronavirus disease (covid-19) pneumonia: a multicenter study. Am J Roentgenol. 2020; 214:1072-7

21. Li K, Wu J, Wu F, Guo D, Chen L, Fang Z, et al. The clinical and chest CT features associated with severe and critical COVID-19 pneumonia. Invest Radiol. 2020;55:327-31.

22. Yang W, Sirajuddin A, Zhang X, Liu G, Teng Z, Zhao S, et al. The role of imaging in 2019 novel coronavirus pneumonia (COVID-19). Eur Radiol. 2020;15:1-9.

23. Santaguida PL, Riley CM, Matchar DB. Assessing risk of bias as a domain of quality in medical test studies. Agency Healthc Res Qual. 2012;12:1-188. 